International Journal of Distributed and Parallel Systems (IJDPS) Vol.2, No.1, January 2011

\title{
Cryptanalysisof two mutual authentication protocols for low-cost RFID
}

\author{
Mohammad Hassan Habibi ${ }^{1}$, Mahmoud Gardeshi ${ }^{2}$, Mahdi Alaghband ${ }^{3}$ \\ ${ }^{1}$ Faculty of Electrical Engineering, I.H. University, Tehran, Iran \\ mohamad.h.habibiegmail.com \\ ${ }^{2}$ Faculty of Electrical Engineering, I.H. University, Tehran, Iran \\ mgardeshi2000@yahoo.com \\ ${ }^{3}$ EEDepartment, Science and Research Campus, Islamic Azad University, Tehran, Iran \\ m. alaghbandesrbiau.ac.ir
}

\begin{abstract}
Radio Frequency Identification (RFID) is appearing as a favorite technology for automated identification, which can be widely applied to many applications such as e-passport, supply chain management and ticketing. However, researchers have found many security and privacy problems along RFID technology. In recent years, many researchers are interested in RFID authentication protocols and their security flaws. In this paper, we analyze two of the newest RFID authentication protocols whichproposed by $\mathrm{Fu}$ et al. and Li et al. from several security viewpoints. We present different attacks such as desynchronization attack and privacy analysis over these protocols
\end{abstract}

\section{KEYWORDS}

RFID; desynchronization;privacy analysis; mutual authentication; security

\section{INTRODUCTION}

Radio Frequency Identification (RFID) technology is one of the most important technologies in this decade. This technology allows identifying the tagging objectives wirelessly using transponders queried by readers through a wireless channel. RFID technology has widely been used in applications such as public transportation [1], supply chain management [2], e-passports [3], location tracking systems [4] and access control systems[5].

There are three main components in a RFID system: tags, readers and a backend server. Each tag contains a microchip, antenna and a certain amount of computational and storage capabilities. A reader queries tags to obtain tag contents through wireless communications and sends this information to the backend server through a secure channel. The backend server is composed of a database and some processors [6]. Since the passive tags have low-cost and low computational capabilities, there are information leakage and many security flaws in passive RFID systems. Inasmuch as the passive tags cannot perform complicated cryptography algorithms. The main threats of a RFID system are as following.

- Tag and reader impersonation: A malicious adversary masquerades as a legitimate tag and tries to use system services by means of reader deception. On the other hand, a legitimate reader is masqueraded by the attacker and he eventually gets access to the stored secrets of $\operatorname{tag}[7]$.

- Man-in-the middle attack: As tags and readers use the wireless channel to communicate each other, so this kind of attack can be occurred. In this situation the attacker intervenes between a legal tag and a legitimate reader and exchanges or modifies the authentication messages [8].

DOI : 10.5121/ijdps.2011.2109 
International Journal of Distributed and Parallel Systems (IJDPS) Vol.2, No.1, January 2011

- Tag tracing and tracking: An adversary traces and tracks legitimate tags from their protocol interactions. The notions untraceability, backward untraceability and forward untraceabilityare related to this attack $[9,10]$.

- Desynchronization: This is an active attack in which a malicious adversary tries to cause the tag and the reader to update inconsistent values and make tag disabled [11].

In recent years, many researchers have tried to propose lightweight and secure authentication protocols $[12,13,14,15,16,17,18,19,20,21,22,23,24]$, but unfortunately many vulnerabilities have been found in their schemes $[25,26,27,28,29,30,31,32,33,34,35,36$, 37, 38]. Recently Fu et al. [39] proposed a scalable RFID mutual authentication and Li et al. [40] suggested a mutual authentication protocol for RFID communication. In this paper, we analyze these protocols and will present three different attacks on FWCFP protocol including desynchronization attack, attack on untraceability in two methods and attack on backward untraceability. Furthermore, one attack is applied on LWJX protocol which is attack on untraceability. The remainder of this paper is organized as following. Related works are studied in section 2.We explain the privacy model for RFID systems in section 3. The FWCFP protocol is summarized as section 4. Our attacks on FWCFP protocol are discussed in section 5. We explain the LWJX protocol in section 6. The security analysis of the LWJX protocol is in section 7 and finally section 8 is assigned to conclusion.The notations in table 1 are used throughout this paper.

TABLE 1.THE NOTATIONS

\begin{tabular}{|c|c|}
\hline A & malicious adversary \\
\hline$E_{K_{s}}()$. & A symmetric encryption function \\
\hline $\mathrm{H}$ & a hash function \\
\hline $\bar{G}$ & a hash function \\
\hline ID & tag identifier \\
\hline IDT & static ID with 96 bit length \\
\hline IDTA & an alias with 96 bit length \\
\hline K & secret value shared by the reader and the tag \\
\hline$K_{S}$ & Secret key only known by the reader \\
\hline $\mathrm{ID}_{\text {old }}$ & ID which is used in current communication by the reader after successful authentication \\
\hline $\mathrm{ID}_{\text {new }}$ & ID which will be used in the new communication by the reader after successful authentication \\
\hline$K_{\text {new }}$ & $\mathrm{K}$ which will be used in the new communication by the reader after successful authentication \\
\hline$K_{\text {old }}$ & $\mathrm{K}$ which is used in current communication by the reader after successful authentication \\
\hline $\mathcal{T}$ & the legitimate tag \\
\hline $\mathrm{R}$ & The legitimate reader and backend server \\
\hline $\mathrm{Rr}$ & random numbers generated by the reader \\
\hline Rt & random numbers generated by the tag \\
\hline $\operatorname{randi}(i=0,1,2)$ & a random number \\
\hline$X_{i}^{j}$ & the item $\mathrm{X}$ related to the $\operatorname{tag} T_{i}$ at time $t_{j}$ \\
\hline
\end{tabular}


International Journal of Distributed and Parallel Systems (IJDPS) Vol.2, No.1, January 2011

\section{RELATED WORKS}

In this section we briefly study some authentication protocols which have been proposed to provide secure communications in RFID systems.

Dimitriou proposed an RFID authentication scheme that uses a challenge-response mechanism [39].Since the tag identifier remains constant between two successful sessions, this protocol is vulnerable to tracking attacks and tag impersonation attack.

In [40], a lightweight authentication protocol is proposed by Ohkubo et. Al. This scheme provides indistinguishability and forward security characteristics. The scheme is based on a hash chain and uses two dissimilar hash functions $H$ and $G$. This protocol does not provide protection against an adversary that tries to de-synchronize the server and the tags, consequently resulting in a DoS attack.

Juels [36] showed that cloning and counterfeiting attacks are applied simply on EPC tags. He proposed an unclonable authentication protocol to solve these problems. However, Duc et al. [20] have presented some weaknesses related to privacy and information leakage in Juels scheme.

In [41], Karthikeyan and Nesterenko suggested a security protocol without complex cryptographic primitives. Only XOR and matrix operations were used in their scheme. Chien and Chen [12] showed that this protocol is vulnerable to replay attacks and does not assure the untraceability property.

A mutual authentication protocol under the EPC C-1 G-2 standard was proposed by Chien and Chen [12]. They had used simple XOR, CRC and PRNG in their scheme. In [12] each tag needs to keep an EPC code and two secret keys $K_{i}, P_{i}$. Secret key $K_{i}$ is used to tag authentication and secret key $P_{i}$ is used to reader authentication. Both $K_{i}$ and $P_{i}$ are updated in each round whereas $E P C$ code is permanent. For each tag secret values $K_{\text {old }}, P_{\text {old }}, K_{\text {new }}, P_{\text {new }}, E P C$ and DATA are stored in database. The protocol is initialed with sending a random number $N_{R}$ by the reader. As a result, the tag replies with $\left(M 1, N_{T}\right)$ where $M 1=C R C\left(E P C\left\|N_{R}\right\| N_{T}\right) \oplus K_{i}$. After receiving the tag's response, the database searches for finding the correct tag and its corresponding information $\left(\left\{K_{\text {old }}, P_{\text {old }}\right\}\right.$ or $\left.\left\{K_{\text {new }}, P_{\text {new }}\right\}\right)$. Thenthe database computes $M 2=\mathrm{CRC}\left(E P C \| N_{T}\right) \oplus P_{x}(x=$ old or new $)$ and sends tag $M 2$. At that point the database updates its secret keys as following: $K_{\text {old }}=K_{\text {new }}, \quad P_{\text {old }}=P_{\text {new }}, \quad K_{\text {new }}=\operatorname{PRNG}\left(K_{\text {new }}\right) \quad$ and $P_{\text {new }}=P R N G\left(P_{\text {new }}\right)$. The tag receives $M 2$ and checks whether $M 2 \oplus P_{i}=C R C\left(E P C \| N_{T}\right)$. If it satisfies, the tag authenticates the database and updates $K_{i}$ and $P_{i}$ the same as with the database, else it terminates the protocol.

Lopez et al. [37] showed some weaknesses of Chien and Chen's protocol including tag and reader impersonation and desynchronization attack. They also showed that this protocol does not guarantee forward security and it is vulnerable to tracing attack. Han and Kwon [14] also presented a desynchronization attack and two tag impersonation attacks on Chien and Chen's protocol in new methods. These attacks were mainly based on weak secure properties of CRC.

\section{RFIDUNTRACEABLE PRIVACY MODEL}

Some privacy models have been proposed by researchers to evaluation of RFID protocols [9, $42,43,44]$. In [42], Juels and Weis gave a formal definition of the privacy and untraceability model. The same definition is described by Ouafi and Phan in their work presented in ISPEC'08 [44] and we will use this model to analyze the SRP protocol. The model that has been described in [44] is summarized as follows. 
International Journal of Distributed and Parallel Systems (IJDPS) Vol.2, No.1, January 2011

The protocol parties are tags $(\mathcal{T})$ and readers $(\mathcal{R})$ which interact in protocol sessions. In this model an adversary $\mathcal{A}$ controls the communication channel between all parties by interacting either passively or actively with them. The adversary $\mathcal{A}$ is allowed to run the following queries:

Execute $(\mathcal{R}, \mathcal{T}, \hat{\imath})$ query. This query models the passive attacks. The adversary $\mathcal{A}$ eavesdrops on the communication channel between $\mathcal{T}$ and $\mathcal{R}$ and gets read access to the exchanged messages between the parties in session $i$ of a truthful protocol execution.

Send $(\mathcal{U}, \mathcal{V}, m, i)$ query. This query models activeattacks by allowing the adversary Ato impersonate some reader $\mathcal{U} \in \mathcal{R}$ (respectively tag $\mathcal{V} \in \mathcal{T}$ ) in some protocol session iand send a message $m$ of its choice to an instance of some tag $\mathcal{V} \in \mathcal{T}$ respectively reader $\mathcal{U} \in \mathcal{R}$ ). Furthermore the adversary $\mathcal{A}$ is allowed to block or alert the message $m$ that is sent from $\mathcal{U}$ to $\mathcal{V}$ (respectively $\mathcal{V}$ to $\mathcal{U}$ ) in session $\boldsymbol{i}$ of a truthful protocol execution.

$\operatorname{Corrupt}\left(\mathcal{T}, K^{\prime}\right)$ query. This query allows the adversary $\mathcal{A}$ to learn the stored secretK of the $\operatorname{tag} \mathcal{T} \in \mathcal{T}$, and which further sets the stored secret to $K^{\prime}$. Corrupt query means that the adversary has physical access to the tag, i.e., the adversary can read and tamper with the tag's permanent memory.

- Test $\left(i, \mathcal{T}_{\mathrm{o}}, \mathcal{T}_{1}\right)$ query. This query does not correspond to any of $\mathcal{A} \mathrm{s}$ abilities, but it is necessary to define the untraceability test. When this query is invoked for session $i$, a random bit $b \in\{0,1\}$ is generated and then, $\mathcal{A}$ is given $\mathcal{T}_{\mathrm{b}} \in\left\{\mathcal{T}_{\mathrm{o}}, \mathcal{T}_{1}\right)$. Informally, $\mathcal{A}$ wins if he can guess the bit $b$.

Untraceable privacy (UPriv) is defined using the game $g$ played between an adversary $\mathcal{A}$ and a collection of the reader and the tag instances. The game gisdivided into three following phases:

Learning phase: $\mathcal{A}$ is given tags $\mathcal{T}_{\mathrm{o}}$ and $\mathcal{T}_{1}$ randomly and he is able to send any Execute, Send and Corrupt queries of its choice to $\mathcal{T}_{0}, \mathcal{T}_{1}$ and reader.

Challenge phase: $\mathcal{A}$ chooses two fresh tags $\mathcal{T}_{0}, \mathcal{T}_{1}$ to be tested and sends a Test $\left(i, \mathcal{T}_{0}, \mathcal{T}_{1}\right)$ query. Depending on a randomly chosen bit $\mathrm{b} \in\{0,1\}, \mathcal{A}$ is given a tag $\mathcal{I}_{\mathrm{b}}$ from the set $\left\{\mathcal{T}_{0}\right.$, $\left.\mathcal{T}_{1}\right\}$. Acontinues making any Execute, and Send queries at will.

Guess phase: finally, $\mathcal{A}$ terminates the game $g$ and outputs a bit $b^{\prime} \in\{0,1\}$, which is its guess of the value of $b$.

The success of $\mathcal{A}$ in winning gamegand thus breaking the notion ofUPrivis quantified in terms Aadvantage in distinguishing whether $\mathcal{A}$ received $\mathcal{T}_{0}$ or $\mathcal{T}_{1}$ and denoted by $\mathbf{A d v}_{\mathbf{A}}^{\text {UPriv }}(\mathrm{k})$ where $\mathrm{k}$ is the security parameter.

$$
\begin{aligned}
& \operatorname{Adv}_{\mathbf{A}}^{\text {UPriv }}(\mathrm{k})=\mid \operatorname{pr}\left(\mathrm{b}=b^{\prime}\right)-\operatorname{pr}(\text { random flip coin })|=| \operatorname{pr}\left(\mathrm{b}^{\prime}=\mathrm{b}\right)-\frac{1}{2} \mid \text { where } \\
& 0 \leq \operatorname{Adv}_{\mathbf{A}}^{\text {UPriv }}(\mathrm{k}) \leq \frac{1}{2} .
\end{aligned}
$$

\section{FWCFPPROTOCOL}

Fu et al. proposed a RFID private mutual authentication in [45]. We summarize the proposed protocol as follows. IDT and K are static ID and key with 96 bit length which are shared between each tag and the reader. Each tag also has an IDTA which is an alias with 96 bit 
International Journal of Distributed and Parallel Systems (IJDPS) Vol.2, No.1, January 2011

length. The reader has a symmetric encryption function $E_{k_{s}}($.$) with secret key K_{s}$ which is known only by it. The reader uses $E_{k_{s}}($.) to encrypt and decrypt IDTA. In each execution of protocol, IDTA is updated as IDTA $=E_{k_{s}}$ (IDT $\|$ rand0) where rand0 is a random number generated by the reader. The steps of the proposed protocol are as following.

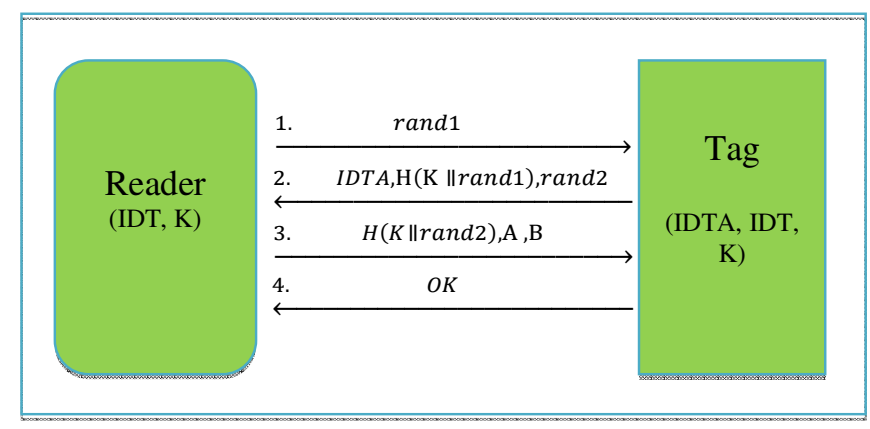

Figure 1. THE FWCFP PROTOCOL

1) The reader generates a random number randl, and sends it to the tag.

2) The reader generates a random number randl, and sends it to the tag.

3 ) The tag generates a random number rand2, computes $\mathrm{H}(\mathrm{K} \|$ rand 1$)$ and sends \{IDTA, H(K \| rand 1 ), rand 2$\}$ to the reader. $\mathrm{H}($.$) is a secure hash function.$

4) The reader decrypts IDTA using the secret key $k_{s}$ to get the permanent ID of tag-IDT, and then retrieves the shared key $\mathrm{K}$ between the tag and the reader by IDT. It computes $\mathrm{H}$ (K ॥ rand 1 ) and checks whether the computed value equals to the received one. If it matches, the tag is authenticated, otherwise the authentication has failed. If the tag is authenticated successfully, the reader generates a new random number $r a n d 0^{\prime}$, computes IDTA ${ }^{\prime}$ as:

$$
\operatorname{IDTA}^{\prime}=E_{k_{s}}\left(\text { IDT } \| \operatorname{rand} 0^{\prime}\right)
$$

Then the reader computes the values A and B as:

$$
\begin{aligned}
& \mathrm{A}=\mathrm{IDTA} \oplus \mathrm{H}(\mathrm{K} \| \text { rand } 1 \| \text { rand } 2) \\
& \mathrm{B}=\mathrm{IDTA} \oplus \mathrm{H}(\mathrm{K} \| \text { rand } 2 \| \text { rand } 1)
\end{aligned}
$$

It also computes $\mathrm{H}(\mathrm{K} \|$ rand 2$)$ and sends ( $\mathrm{H}(\mathrm{K} \|$ rand2), $\mathrm{A}, \mathrm{B})$ to the tag.

5) The tag checks $\mathrm{H}(\mathrm{K} \|$ rand2) to authenticate the reader. If it matches, the reader is authenticated; otherwise the whole authentication has failed. If the reader is authenticated successfully, the tag computes $\mathrm{H}(\mathrm{K} \|$ rand $1 \|$ rand 2$)$ and $\mathrm{H}(\mathrm{K} \|$ rand $2 \|$ randl). Then it computes two new aliases as:

$$
\begin{aligned}
& \mathrm{IDTA} 1=\mathrm{A} \oplus \mathrm{H}(\mathrm{K} \| \text { rand } 1 \| \text { rand } 2) \\
& \text { IDTA2 }=\mathrm{B} \oplus \mathrm{H}(\mathrm{K} \| \text { rand } 2 \| \text { rand } 1)
\end{aligned}
$$

If IDTA1 $=$ IDTA2, the tag stores IDTA1 as the new alias IDTA and sends OK to the reader.

\section{SECURITY ANALYSIS OF THE FWCFP PROTOCOL}

In this section, we analyze the FWCFP protocol [45] from the security point of view. We have found many security vulnerabilities in this protocol,so we present four different attacks on synchronization and untraceability of this protocol. 
International Journal of Distributed and Parallel Systems (IJDPS) Vol.2, No.1, January 2011

\subsection{Attack on Synchronization}

We have found a fundamental weakness in this protocol. An attacker can exploit from this weakness and desynchronize a legal tag $\mathcal{T}_{\mathrm{i}}$ and the legitimate reader. The procedure of the attack is as following.

1) The adversary eavesdrops a valid session between the legal tag $\mathcal{T}_{\mathrm{i}}$ and the reader. He lets parties send the first and the second message safely, but he changes the third message and modifies the values $\mathrm{A}, \mathrm{B}$ to $\mathrm{A}^{\prime}, B^{\prime}$ as:

$$
\begin{aligned}
& A^{\prime}=\mathrm{A} \oplus \mathrm{IDTA}^{\prime} \\
& B^{\prime}=\mathrm{B} \oplus \mathrm{IDTA}^{\prime}
\end{aligned}
$$

where IDTA $\square$ is an arbitrary bit string with 96 bit length. Then the adversary sends (A', B', $\mathrm{H}(\mathrm{K} \| \mathrm{rand} 2))$ to $\mathrm{Ti}$ as the third message.

2) Upon receiving the third message, $\mathcal{T}_{\mathrm{i}}$ computes $\mathrm{H}(\mathrm{K} \|$ rand2), checks whether the computed value equals to the received one. Because it matches, $\mathcal{T}_{\mathrm{i}}$ authenticates the adversary and computes IDTA1, IDTA2 as:

$\mathrm{IDTA} 1=\mathrm{A}^{\prime} \oplus \mathrm{H}(\mathrm{K} \|$ rand $1 \|$ rand 2$)=\mathrm{A} \oplus \operatorname{IDTA} \oplus \mathrm{H}(\mathrm{K} \|$ rand $1 \|$ rand 2$)=\mathrm{IDTA} \oplus \mathrm{H}(\mathrm{K}$ $\|$ rand $1 \|$ rand 2$) \oplus \mathrm{IDTA} \mathrm{A}^{\prime} \oplus \mathrm{H}(\mathrm{K} \|$ rand $1 \|$ rand 2$)=\mathrm{IDTA} \oplus \mathrm{IDTA}^{\prime \prime}$

$$
\begin{gathered}
\mathrm{IDTA} 2=\mathrm{B}^{\prime} \oplus \mathrm{H}(\mathrm{K} \| \text { rand } 2 \| \text { rand } 1)=\mathrm{B} \oplus \mathrm{IDTA} \mathrm{A}^{\prime} \oplus \mathrm{H}(\mathrm{K} \| \text { rand } 2 \| \text { rand } 1)=\mathrm{IDTA} \oplus \mathrm{H}(\mathrm{K} \| \\
\text { rand2 } \| \text { rand } 1) \oplus \mathrm{IDTA} \oplus \mathrm{H}(\mathrm{K} \| \text { rand } 2 \| \text { rand } 1)=\mathrm{IDTA} \mathrm{I}^{\prime} \oplus \mathrm{IDT} \AA^{\prime \prime}
\end{gathered}
$$

Because IDTA1 $=$ IDTA2, $\mathcal{T}_{\mathrm{i}}$ updates the stored IDTA as:

$$
\text { IDTA }=\text { IDTA1 }=\text { IDTÁ } \oplus I D T A ́
$$

At the next sessions, whenever $\mathcal{T}_{\mathrm{i}}$ wants to authenticate itself to the reader, it sends IDTA $\square \oplus$ IDTA $\square$ to the reader. After decryptionIDTA $\square \oplus$ IDTA $\square$, the reader extracts IDT' which is not equal to IDT, so the reader does not find IDT' in its database, therefore the reader always rejects $\mathcal{T}_{\mathrm{i}}$ and they have no way to resynchronization.

\subsection{Attack on Untraceability}

A main weakness in designing this protocol is the fact that the term $\mathrm{H}$ (shared key $\|$ a random number) has the same structure in the second and the third flow of the protocol. An adversary can exploit this weakness and trace a tag as following.

Learning phase:The adversary is given tag $\mathcal{T}_{0}$ at random. A eavesdrops a perfect session between $\mathcal{T}_{0}$ and a legitimate reader. He gets the values rand2 and $\mathrm{H}\left(\mathrm{K}_{0} \|\right.$ rand2) from the first and the second flows of the protocol respectively by an Execute query. He reserves these values.

Challenge phase: $\mathrm{A}$ is giventag $\mathcal{T}_{\mathrm{b}} \in\left\{\mathcal{T}_{0}, \mathcal{T}_{1}\right\}$ randomly. He starts a new session with $\mathcal{T}_{\mathrm{b}}$ and sends rand 2 to it as the first message by Send query. $\mathcal{T}_{\mathrm{b}}$ responds with: 
International Journal of Distributed and Parallel Systems (IJDPS) Vol.2, No.1, January 2011

$\left(\mathrm{H}\left(\mathrm{K}_{\mathrm{b}} \| \operatorname{rand} 2\right)\right.$, IDTA, rand'2) and the adversary reserves $\mathrm{H}\left(\mathrm{K}_{\mathrm{b}} \| \operatorname{rand} 2\right)$.

Guess phase: If $\mathrm{H}\left(\mathrm{K}_{\mathrm{b}} \|\right.$ rand2 $)=\mathrm{H}\left(\mathrm{K}_{0} \|\right.$ rand2), the adversary outputs $b^{\prime}=0$ and guesses $\mathcal{T}_{0}$, otherwise he outputs $b^{\prime}=1$ and guesses $\mathcal{T}_{1}$. The advantage of the adversary is:

$\boldsymbol{A d v _ { \boldsymbol { A } } ^ { \text { Upriv } }}(\boldsymbol{k})=\mid \operatorname{pr}($ A wins $)-\operatorname{pr}($ random coin flip $)|=| \operatorname{pr}\left(b^{\prime}=\mathrm{b}\right)-\frac{1}{2}|=|\left(1-2^{-n}\right)-$ $\frac{1}{2} \mid=\frac{1}{2}-2^{-n}$ Where $|\mathrm{H}()|=.\mathrm{n}$

By having $\mathrm{H}\left(\mathrm{K}_{0} \|\right.$ rand2), if $\mathcal{T}_{\mathrm{b}}=\mathcal{T}_{0}$, then with the probability of 1 we have $\mathrm{H}\left(\mathrm{K}_{\mathrm{b}} \|\right.$ rand2 $)=$ $\mathrm{H}\left(\mathrm{K}_{0} \|\right.$ rand2), but if $\mathcal{T}_{\mathrm{b}}=\mathcal{T}_{1}$, then with the probability of $2^{-n}$ we have $\mathrm{H}\left(\mathrm{K}_{\mathrm{b}} \|\right.$ rand2 $)=\mathrm{H}\left(\mathrm{K}_{0} \|\right.$ rand2), because $\mathrm{H}($.) is a bit string with length $\mathrm{n}$.

\subsection{Attack on Backward Untraceability}

We use the notion backward untraceability from [8] and use the privacy model from [44] to show that the FWCFP protocol doesn't assure the backward untraceability.

Learning phase: $\mathrm{A}$ is given tag $\mathcal{T}_{0}$ at random, he sends $\mathcal{T}_{0}$ Corrupt query at time $\mathrm{t}_{1}$ and gets the secrets of the $\mathcal{T}_{0}$ at time $\mathrm{t}_{1}$ as $\left(K_{0}^{1}, I D T_{0}^{1}, I D T A_{0}^{1}\right)$.

Challenge phase:The adversary is given $\mathcal{T}_{\mathrm{b}} \in\left\{\mathcal{T}_{0}, \mathcal{T}_{1}\right\}$ randomly. He can have access to the previous session accomplished between $\mathcal{T}_{\mathrm{b}}$ and Rat time $\mathrm{t}_{0}<\mathrm{t}_{1}$. He gets randl and $\mathrm{H}\left(\mathrm{K}_{\mathrm{b}} \|\right.$ randl) by Execute query.

Guess phase: Because the secret key of $\mathcal{T}_{0}$ is fixed, we have $K_{0}^{1}=K_{0}^{0}$. The adversary also has randl from the session accomplished at time $\mathrm{t}_{0}$. So he can compute $\mathrm{H}\left(\mathrm{K}_{0} \|\right.$ randl $)$. Now, if $\mathrm{H}\left(\mathrm{K}_{\mathrm{b}} \|\right.$ rand 1$)=\mathrm{H}\left(\mathrm{K}_{0} \|\right.$ rand 1$)$, he outputs $b^{\prime}=0$ and guesses $\mathcal{T}_{0}$, otherwise he outputs $b^{\prime}=1$ and guesses $\mathcal{T}_{1}$. The advantage of the adversary is:

$$
\begin{aligned}
& \boldsymbol{A d v _ { A } ^ { \text { Upriv } }}(\boldsymbol{k})=\mid \operatorname{pr}(\text { A wins })-\operatorname{pr}(\text { random coin flip })|=| \operatorname{pr}\left(b^{\prime}=\mathrm{b}\right)-\frac{1}{2} \mid= \\
& \left|\left(1-2^{-n}\right)-\frac{1}{2}\right|=\frac{1}{2}-2^{-n} \operatorname{Where}|\mathrm{H}(.)|=\mathrm{n}
\end{aligned}
$$

Because the adversary can compute $\mathrm{H}\left(\mathrm{K}_{0} \|\right.$ rand1), he owns this value. By having $\mathrm{H}\left(\mathrm{K}_{0} \|\right.$ rand1), if $\mathcal{T}_{\mathrm{b}}=\mathcal{T}_{0}$, then with the probability of 1 we have $\mathrm{H}\left(\mathrm{K}_{\mathrm{b}} \|\right.$ randl $)=\mathrm{H}\left(\mathrm{K}_{0} \|\right.$ randl $)$, but if $\mathcal{T}_{\mathrm{b}}=\mathcal{T}_{1}$, then with the probability of $2^{-n}$ we have $\mathrm{H}\left(\mathrm{K}_{\mathrm{b}} \|\right.$ rand 1$)=\mathrm{H}\left(\mathrm{K}_{0} \|\right.$ rand 1$)$, because $\mathrm{H}$ (.) is a bit string with length $n$.

\section{LWJX PROTOCOL}

Li et al. proposed an authentication protocol for secure RFID communication [46]. The proposed protocol is as it follows.Each tag stores an initial ID and a secret key $\mathrm{K}$ shared by the tag and the reader. The reader keeps the following information for each tag: ID with initial value same as to tag's ID, hash value of $\mathrm{ID}_{\text {new }}$ with the initial value of $\mathrm{H}$ (ID), hash value ofID old with the initial value is empty, new value of secret key $\mathrm{K}_{\text {new }}$ with the initial value of $\mathrm{K}$, old value of secret key $\mathrm{K}_{\text {old }}$ with the initial value is empty. The parameter $\mathrm{M}$ holds howmany times a tag has had unsuccessful sessions. Two hash functions $\mathrm{H}$ and $\mathrm{G}$ are implemented on each tag and on the reader. The procedure of authentication is as follows.Each tag stores an initial ID and a secret key $\mathrm{K}$ shared by the tag and the reader. The reader keeps the following information 
International Journal of Distributed and Parallel Systems (IJDPS) Vol.2, No.1, January 2011

for each tag: ID with initial value same as to tag's ID, hash value of $\mathrm{ID}_{\text {new }}$ with the initial value of $\mathrm{H}$ (ID), hash value ofID old $_{\text {with }}$ the initial value is empty, new value of secret key $\mathrm{K}_{\text {new }}$ with the initial value of $\mathrm{K}$, old value of secret key $\mathrm{K}_{\text {old }}$ with the initial value is empty. The parameter $\mathrm{M}$ holds howmany times a tag has had unsuccessful sessions. Two hash functions $\mathrm{H}$ and $\mathrm{G}$ are implemented on each tag and on the reader. The procedure of authentication is as follows.

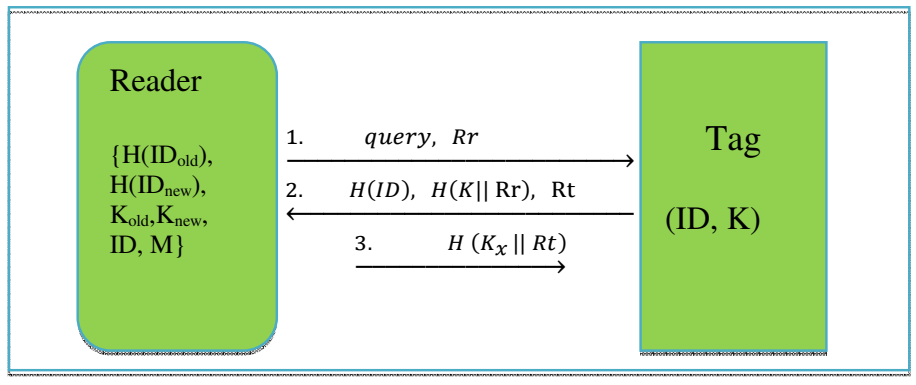

Figure 2. THE LWJX PROTOCOL

1) The reader generates a number $\mathrm{Rr}$ at random and sends it to the tag.

2) After receiving $\mathrm{Rr}$, the tag generates a number Rt at random, computes $\mathrm{H}$ (ID) and $\mathrm{H}$ (K ॥ $\mathrm{Rr}$ ), then it sends them and Rt to the reader.

3) The reader searches in the database records tofind whether there is a $\mathrm{H}\left(\mathrm{ID}_{\text {old }}\right), \mathrm{H}\left(\mathrm{ID}_{\text {new }}\right)$ equal to the received $H$ (ID). Three possible cases occur:

a) If no value is found, it terminates the protocol.

b) If it is found that $\mathrm{H}\left(\mathrm{ID}_{\text {new }}\right)=\mathrm{H}$ (ID), then the readercomputes $\mathrm{H}\left(\mathrm{K}_{\text {new }} \| \mathrm{Rr}\right)$ and compares it with the received $\mathrm{H}(\mathrm{K} \| \mathrm{Rr})$, if $\mathrm{H}\left(\mathrm{K}_{\text {new }} \| \mathrm{Rr}\right) \neq \mathrm{H}(\mathrm{K} \| \mathrm{Rr})$, then $\mathrm{R}$ terminates the protocol; otherwise, Rresets $M=0$, computes $H\left(K_{\text {new }} \| R t\right)$ and sends $H\left(K_{\text {new }} \| R t\right)$ to the tag. Finally, the reader updates its secret values as following:

$$
\begin{aligned}
& \mathrm{ID}=\mathrm{G}(\mathrm{ID}) \\
& \mathrm{H}\left(\mathrm{ID}_{\text {old }}\right)=\mathrm{H}\left(\mathrm{ID}_{\text {new }}\right) \\
& \mathrm{H}\left(\mathrm{ID}_{\text {new }}\right)=\mathrm{H}(\mathrm{ID}) \\
& \mathrm{K}_{\text {old }}=\mathrm{K}_{\text {new }} \\
& \mathrm{K}_{\text {new }}=\mathrm{ID} \oplus \mathrm{Rr} \oplus \mathrm{Rt}
\end{aligned}
$$

c) If $\mathrm{H}\left(\mathrm{ID}_{\text {old }}\right)=\mathrm{H}(\mathrm{ID})$, first the reader checks $\mathrm{M}$, if $\mathrm{M}$ isgreater than the upper limit, it terminates the protocol and issues a warning; otherwise it makes $M=m+1$, now if $\mathrm{H}\left(\mathrm{K}_{\text {old }} \| \mathrm{Rr}\right) \neq \mathrm{H}(\mathrm{K} \| \mathrm{Rr})$, then $\mathrm{R}$ terminates the communication; otherwise, Rcomputes $\mathrm{H}\left(\mathrm{K}_{\text {old }} \| \mathrm{Rt}\right)$ and sends $\mathrm{H}\left(\mathrm{K}_{\text {old }} \| \mathrm{Rt}\right)$ to the tag. The reader doesn't update in this case.

4) After receiving $\mathrm{H}\left(\mathrm{K}_{\mathrm{x}} \| \mathrm{Rt}\right)\{\mathrm{x}=$ old or new $\}$, ifH $\left(\mathrm{K}_{\mathrm{x}} \| \mathrm{Rt}\right)=\mathrm{H}(\mathrm{K} \| \mathrm{Rt})$, the tagupdates its secretvalues as:

$$
\begin{aligned}
& \mathrm{ID}=\mathrm{G}(\mathrm{ID}) \\
& \mathrm{K}=\mathrm{ID} \oplus \mathrm{Rr} \oplus \mathrm{Rt}
\end{aligned}
$$

\section{PRIVACY ANALYSIS OF LWJX PROTOCOL}

In this section, we analyze the LWJX protocol and give an attack on this protocol. 


\subsection{Attack on Untraceability}

We give our privacy analysis on LWJX protocol according to the privacy model discussed in [18] which has been explained it in section III. We show that the LWJX protocol does not have untraceability.

Learning phase: The adversary is given tag $\mathcal{T}_{0}$ at random. He masquerades as a legitimate reader and starts a new session with tag $\mathcal{T}_{0}$ by sending Send query. He sends $\operatorname{Rr}_{1}$ to $\mathcal{T}_{0}$ and gets its response as $\left(\mathrm{H}\left(I D_{0}\right), \mathrm{H}\left(\mathrm{K}_{0} \| \mathrm{Rr}_{1}\right), \mathrm{Rt}\right)$. The adversary reserves these values and terminates the session to avoid the $\mathcal{T}_{0}$ updating.

Challenge phase: $\mathrm{A}$ is given $\mathcal{T}_{\mathrm{b}} \in\left\{\mathcal{T}_{0}, \mathcal{T}_{1}\right\}$ randomly. The adversary performs a new session with $\mathcal{T}_{\mathrm{b}}$ by sending Send query. He sends $\mathrm{Rr}_{1}$ to $\mathcal{T}_{\mathrm{b}}$ and gets its response as $\left(\mathrm{H}\left(I D_{b}\right), \mathrm{H}\left(\mathrm{K}_{\mathrm{b}} \|\right.\right.$ $\left.\left.\mathrm{Rr}_{1}\right), R t^{\prime}\right)$. The adversary reserves these values and terminates the session.

Guess phase: The adversary can guess the correct tag in two ways:

If $\mathrm{H}\left(\mathrm{ID}_{\mathrm{b}}\right)=\mathrm{H}\left(\mathrm{ID}_{0}\right)$, then the adversary outputs $\mathrm{b}^{\prime}=0$ and guesses $\mathcal{T}_{0}$; otherwise he outputs $\mathrm{b}^{\prime}=1$ and guesses $\mathcal{T}_{1}$.

1) If $\mathrm{H}\left(\mathrm{K}_{\mathrm{b}} \| \mathrm{Rr}_{1}\right)=\mathrm{H}\left(\mathrm{K}_{0} \| \mathrm{Rr}_{1}\right)$, then the adversary outputs $\mathrm{b}^{\prime}=0$ and guesses $\mathcal{T}_{0}$, otherwise he outputs $\mathrm{b}^{\prime}=1$ and guesses $\mathcal{T}_{1}$. In both cases, A wins with high probability:

$$
\begin{aligned}
& \boldsymbol{A d v _ { A } ^ { U p r i v }}(\mathrm{k})=\mid \operatorname{pr}(A \text { wins })-\operatorname{pr}(\text { random coin flip })|=| \operatorname{pr}\left(b^{\prime}=\mathrm{b}\right)-\frac{1}{2} \mid= \\
& \left|\left(1-2^{-n}\right)-\frac{1}{2}\right|=\frac{1}{2}-2^{-n} \text { Where }|\mathrm{H}(.)|=\mathrm{n}
\end{aligned}
$$

Because $|\mathrm{H}()|=.\mathrm{n}$, we have $\mathrm{H}\left(\mathrm{ID}_{0}\right)=\mathrm{H}\left(\mathrm{ID}_{1}\right)$ with the probability of $2^{-n}$, so the adversary can guess the correct tag with the probability of $1-2^{-n}$.

\section{CONCLUSION}

In this paper, we showed some security and privacy vulnerabilities of the RFID authentication protocols proposed by $\mathrm{Fu}$ et al [45] and $\mathrm{Li}$ et al [46]. We also present the desynchronization attack and tag tracing on [45]. In desynchronization attack, an adversary can easily change the third message transmitted in protocol and desynchronize the target tag and the legitimate reader. We also presented the privacy analysis of this protocol in a formal privacy model. We showed the FWCFP protocol doesn't assure untraceability, backward untraceability and forward untraceability. We also presented some attacks on privacy and anonymity of [46]. It has shown that untraceability and forward untraceability aren't assured by this protocol.

\section{ACKNOWLEDGMENT}

This work is supported by the Education \& Research Institute for ICT, Tehran, Iran.

\section{REFERENES}

[1] Transport for London, Oyster card, http://www.oystercard.co.uk.

[2] "Michelin Embeds RFID Tags in Tires", RFID Journal, http://www.rfidjournal.com/article/ articleview/ 269 /1/1/. Accessed 17 Jan 2003 
International Journal of Distributed and Parallel Systems (IJDPS) Vol.2, No.1, January 2011

[3] Hoepman, J.-H.,Hubbers, E., Jacobs, B., Oostdijk, M., Scherer, R.W., "Crossing borders: Security and privacy issues of the European e-passport", NAME (IWSEC 2006). LNCS, Springer-Heidelberg, vol. 4266 ( 2006) 152-167.

[4] Li, Z., Chu C.-H., and Yao, W., "SIP-RLTS: An RFID location tracking system based on SIP", In: Proceedings of the 2008 IEEE International Conference on RFID, 2008, pp. 173-182.

[5] E.-C. Australia, "Access control, sensor control, and trans-ponders", at: http://www.rfid. com.au/rfid uhf.htm,2008.

[6] A. Juels, "RFID security and privacy: a research survey", Selected Areas in Communications 24 (2) (2006) 381-394. February.

[7] Van Deursen, T., Radomirovic, S., "Attacks on RFID protocols", Cryptology ePrint Archive, Report 2008/310, 2008. <http://eprint.iacr.org/>.

[8] H. Gilbert, M. Robshaw, H. Sibert, "An active attack against HB+ - A provably secure lightweight authentication protocol", Cryptology ePrint Archive, http://eprint.iacr.org/2005/ 23. pdf.

[9] Lim, C.H., and Kwon, T., "Strong and robust RFID authentication enabling perfect ownership transfer", In Proceedings of ICICS '06, LNCS 4307 (2006) 1-20

[10] R. Phan, "Cryptanalysis of a new ultralightweight RFID authentication protocol-SASI", IEEE Transactions on Dependable and Secure Computing 6(4): Oct.-Dec. (2009) 316-320.

[11] Peris-Lopez, P., Hernandez-Castro, J.C., Estevez-Tapiador, J.M., and Ribagorda, A., "Vulnerability analysis of RFID protocols for tag ownership transfer", Computer Networks 54 (2010) 1502-1508

[12] Chien, H., Chen, C., "Mutual Authentication Protocol for RFID Conforming to EPC Class1 Generation-2 Standards", Computer Standards \& Interfaces, 29 (2007) 254-259

[13] Indumathi, G., and Murugesan, K., "A bandwith efficient scheduling framework for non real time applications in wireless networks", International Journal of Distributed and Parallel systems (IJDPS) Vol.1, No.1, September 2010.

[14] Han, D., Kwon, D.: Vulnerability of an RFID authentication protocol conforming to EPC Class-1Generation-2 Standards. Computer Standards \& Interfaces 31 (2009) 648-652.

[15] Peris-Lopez, P., Hernandez-Castro, J.C., Estevez-Tapiador, J.M, and Ribagorda, A., "LMAP: A real lightweight mutual authentication protocol for low-cost RFID tags", In: Proceedings of the 2nd Workshop on RFID Security, July 2006.

[16] Yeh, T.-C., Wang, Y.-J., Kuo, T.-C., Wang, S.-S., "Securing RFID systems conforming to EPC Class-1 Generation-2 standard", Expert Systems with Applications 37 (2010) 7678-7683

[17] P. Peris-Lopez, J. C. Hernandez-Castro, J. M. Estevez-Tapiador, and A. Ribagorda, "EMAP: An efficient mutual authentication protocol for low-cost RFID tags", In Proc. of IS'06, volume 4277 of LNCS, pages 352-361, Springer-Verlag, 2006.

[18] Gu, Y., Wu, W., "Mutual authentication protocol based on tag ID number updating for low-cost RFID", In Proceedings of the first IEEE International Conference on Network Infrastructure and Digital Content(IC-NIDC2009), pp. 548-551, 2009.

[19] Kim, K. H., Choi, E. Y., Lee, S. M., and Lee, D.H., "Secure EPCglobal Class-1 Gen-2 RFID system against security and privacy problems", In Proc. of OTM-IS'06, volume 4277 of LNCS, pages 362-371. Springer-Verlag, 2006.

[20] Duc, D.N., Park, J., Lee, H., and Kwangjo, K., "Enhancing security of epcglobal Gen-2 RFID tag against traceability and cloning", In Proc. of Symposium on Cryptography and Information Security, 2006.

[21] Li T., and Wang, G., "SLMAP-A secure ultra-lightweight rfid mutual authentication protocol", Proc. of Chinacrypt'07, 2007.

[22] Kulseng, L., Yu, Z., Wei, Y., and Guan, Y., "Lightweight mutual authentication and ownership transfer for RFID Systems", In Proceedings of IEEE INFOCOM 2010, 1-5, CA, March (2010).

[23] Song, B., and Mitchell, C. J., "RFID authentication protocol for low-cost tags", In Wisec 2008, pages 140-147. 
International Journal of Distributed and Parallel Systems (IJDPS) Vol.2, No.1, January 2011

[24] Chien, H. Y., "SASI: A new ultralightweightrfid authentication protocol providing strong authentication and strong integrity", IEEE Transactions on Dependable and Secure Computing, 4(4):337-340, 2007.

[25] Peris-Lopez, P., Li, T., Lim, T.-L., Hernandez-Castro, J. C., Estevez- Tapiador, J. M., and Ribagorda, A., "Vulnerability analysis of a mutual authentication scheme under the epc class-1 generation-2 standard", In Hand. of RFIDSec'08, 2008

[26] Rizomiliotis, P., Rekleitis, E., Gritzalis, S.,"Security analysis of the Song- Mitchell authentication protocol for low-cost RFID tags", Communications Letters, IEEE 13 (4) (2009), pp. 274-276..

[27] Lin, C.-L., and Chang, G.-G., "Cryptanalysis of EPC class 1 generation 2 RFID authentications", Information Security Conference 2007, ChiaYi, Taiwan.

[28] Peris-Lopez, P., Hernandez-Castro, J. C., Estevez-Tapiador, J. M., and Ribagorda, A., "Practical attacks on a mutual authentication scheme under the EPC Class-1 Generation-2 standard", Computer Communications 32 (2009) 1185-1193.

[29] Habibi, M. H., Gardeshi, M., Alaghband, M., " Cryptanalysis of a mutual authentication protocol for low-cost RFID”, In proceedings of IEEE International Conference on Intelligent Information Networks (ICIIN 2011), UAE, 2011.

[30] Van Deursen, T., Radomirovi'c, S., "Security of RFID protocols - A case study", Electronic Notes in Theoretical Computer Science 244 (2009) 41-52.

[31] Habibi, M. H., Gardeshi, M., Alaghband, M., "Practical Attacks on a RFID Authentication Protocol Conforming to EPC C-1 G-2 Standard", International Journal of Ubicomp, Volume 2, Number 1,(2011)

[32] Habibi, M. H., Gardeshi, M., Alaghband, M., "Security analysis of an RFID mutual authentication protocol for RFID systems", In proceedings of IEEE International Conference on Intelligent Information Networks (ICIIN 2011), UAE, 2011.

[33] Habibi, M. H., Gardeshi, M., Alaghband, M., "Attacks and improvements to a new RFID mutual Authentication protocol", In proceedings of Third Workshop on RFID Security: RFIDsec Asia 2011, China, 2011.

[34] Li, T., Deng, R.H., "Vulnerability analysis of EMAP-An efficient RFID mutual authentication protocol", In: AReS 2007: Second International Conference on Availability, Reliability and Security (2007).

[35]Alomair, B., Lazos, L., and Poovendran, R., "Passive attacks on a class of authentication protocols for RFID”, K.-H. Nam and G. Rhee (Eds.): ICISC 2007, LNCS 4817, pp. 102-115, 2007.

[36] Juels, A., "Minimalist cryptography for low-cost RFID tags", In Proc. of SCN'04, volume 3352 of LNCS, pp. 149-164, Springer-Verlag, 2004.

[37] Peris-Lopez, P., Li, T., Lim, T.-L., Hernandez-Castro, J. C., Estevez- Tapiador, J. M., and Ribagorda, A.,, "Cryptanalysis of a novel authentication protocol conforming to EPC-C-1 G-2 standard", Computer Standards \& Interfaces, Elsevier Science Publishers, doi:10.1016/j.csi. 2008.05.012, 2008.

[38] Han, D., Kwon, D.: Vulnerability of an RFID authentication protocol conforming to EPC Class-1Generation-2 Standards. Computer Standards \& Interfaces 31 (2009) 648-652.

[39] Dimitriou, T., "A lightweight RFID protocol to protect against traceability and cloning attacks", In SecureComm, pages 59-66, 2005.

[40] Ohkubo, M., Suzuki, K., Kinoshita, S., "Cryptographic approach to "privacy-friendly tags", In 2003 MIT RFID Privacy Workshop, 2003.

[41] Karthikeyan, S., Nesterenko, M., "RFID security without extensive cryptography", In Proc. of SASN '05, ACM (2005) 63-67.

[42] Juels, A., and Weis, S.A., "Defining strong privacy for RFID”, In Proceedings of PerCom '07 (2007) 342-347, http://eprint.iacr.org/2006/137.

[43] Avoine, G., "Adversarial model for radio frequency identification", Cryptology ePrint Archive, report 2005/049. http://eprint.iacr.org/2005/049. 
International Journal of Distributed and Parallel Systems (IJDPS) Vol.2, No.1, January 2011

[44] Ouafi, K., and Phan, R.C.-W. "Privacy of recent RFID authentication protocols", L. Chen, Y. Mu, and W. Susilo (Eds.): ISPEC 2008, LNCS 4991, pp. 263-277, 2008.

[45] Fu, J., Wu, C., Chen, X., Fan, R., and Ping, L., "Scalable pseudo random RFID private mutual authentication", 2nd IEEE International Conference on Computer Engineering and Technology (ICCET). V. 7, pp. 497-500, China, 2010.

[46] J. Li, Y. Wang, B. Jiao and Y. Xu,"An Authentication Protocol for Secure and Efficient RFID Communication”, In: Proceedings of 2010 International Conference on Logistics Systems and Intelligent Management(ICLSIM), Harbin, China, January, 2010,pp. 1648-1651.

\section{Authors}

Mohammad Hassan Habibi received the Bachelor's degree in Telecommunication Engineering From Kerman University, Kerman, Iran, in 2007 and Master's degree in Telecommunication in the field of Cryptography with the honor degree from IHU, Tehran, Iran (2011), where he obtained the Best Student Academic Award.

Currently, he is a research assistant (RA) at the research center of cryptography, IHU, Tehran, Iran. His research interest includes: Lightweight cryptography, RFID security, authentication protocols,cryptanalysis, public key cryptography and lightweight primitives.

Mahmoud Gardeshi received his Erudition Degree in applied mathematics from Amir KabirUniversity, Islamic Republic of Iran in 2000. Currently, he is a researcher at the I. H. University. His research interest includes: cryptography and information security.

Mahdi R. Alaghband received his B.S. degree in Electrical engineering in 2005 and M.S. degree in Communications, Cryptology \& Information Security in 2008. Currently, he is both a Ph.D. candidate at the department of Electrical and Computer Engineering, Azad University and research assistant on Information Systems and Security Lab (ISSL), EE Dept., Sharif University of Technology. His research interests include authentication protocol especially in RFID systems, security in wireless sensor networks and

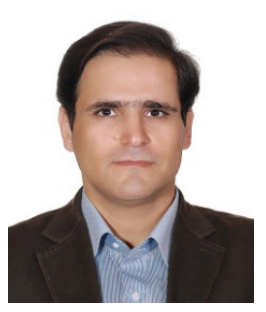
lightweight cryptographic. 